\title{
Study of Nanotechnology and Its Application
}

\author{
Dr.Sumit Kumar Gupta
}

Dean, Faculty of science, Department of Physics , Parishkar college of Global Excellence, Jaipur

\begin{abstract}
Nanotechnology is new frontiers of this century. The world is facing great challenges in meeting rising demands for basic commodities(e.g., food, water and energy), finished goods (e.g., cellphones, cars and airplanes) and services (e.g., shelter, healthcare and employment) while reducing and minimizing the impact of human activities on Earth's global environment and climate. Nanotechnology has emerged as a versatile platform that could provide efficient, cost-effective and environmentally acceptable solutions to the global sustainability challenges facing society. In recent years there has been a rapid increase in nanotechnology in the fields of medicine and more specifically in targeted drug delivery. Opportunities of utilizing nanotechnology to address global challenges in (1) water purification, (2) clean energy technologies, (3) greenhouse gases management, (4) materials supply and utilization, and (5) green manufacturing and chemistry. Smart delivery of nutrients, bio-separation of proteins, rapid sampling of biological and chemical contaminants, and nano encapsulation of nutraceuticals are some of the emerging topics of nanotechnology for food and agriculture. Nanotechnology is helping to considerably improve, even revolutionize, many technology and Industry sectors: information technology, energy, environmental science, medicine, homeland security, food safety, and transportation, among many others. Today's nanotechnology harnesses current progress in chemistry, physics, materials science, and biotechnology to create novel materials that have unique properties because their structures are determined on the nanometer scale. This paper summarizes the various applications of nanotechnology in recent decades Nanotechnology is one of the leading scientific fields today since it combines knowledge from the fields of Physics, Chemistry, Biology, Medicine, Informatics, and Engineering. It is an emerging technological field with great potential to lead in great breakthroughs that can be applied in real life. Novel Nano and biomaterials, and Nano devices are fabricated and controlled by nanotechnology tools and techniques, which investigate and tune the properties, responses, and functions of living and non-living matter, at sizes below100 $\mathrm{nm}$. The application and use of Nano materials in electronic and mechanical devices, in optical and magnetic components, quantum computing, tissue engineering, and other biotechnologies, with smallest features, widths well below $100 \mathrm{~nm}$, are the economically most important parts of the nanotechnology nowadays and presumably in the near future. The number of Nano products is rapidly growing since more and more Nano engineered materials are reaching the global market the continuous revolution in nanotechnology will result in the fabrication of nanomaterial with properties and functionalities which are going to have positive changes in the lives of our citizens, be it in health, environment, electronics or any other field. In the energy generation challenge where the conventional fuel resources cannot remain the dominant energy source, taking into account the increasing consumption demand and the $\mathrm{CO}_{2}$.Emissions alternative renewable energy sources based on new technologies have to be promoted. Innovative solar cell technologies that utilize nanostructured materials and composite systems such as organic photovoltaic offer great technological potential due to their attractive properties such as the potential of large-scale and low-cost roll-to-roll manufacturing processes.
\end{abstract}

Corresponding author: Dr. Sumit Kumar Gupta, Department of Physics, Dean, Faculty of science, Parishkar college of Global Excellence, Jaipur, Email: sumitguptagit@gmail.com 
Received: March 03, 2020; Accepted: March 11, 2020 ; Published: March 13, 2020 ;

Keywords: Nanotechnology, Nanomaterial, drug delivery

\section{Introduction}

The term nanotechnology comes from the combination of two words: the Greek numerical prefix Nano referring to a billionth and the word technology. As an outcome, Nanotechnology or Nano scaled Technology is generally considered to be at a size below 0:1_m or $100 \mathrm{~nm}$ (a nanometer is one billionth of a meter, 10_9 m). Nano scale science (or Nano science) studies the phenomena, properties, and responses of materials at atomic, molecular, and macromolecular scales, and in general at sizes between 1 and $100 \mathrm{~nm}$. In this scale, and especially below $5 \mathrm{~nm}$, the properties of matter differ significantly (i.e., quantumscale effects play an important role) from that at a larger particulate scale. Nanotechnology is then the design, the manipulation, the building, the production and application, by controlling the shape and size, the properties-responses and functionality of structures, and devices and systems of the order or less than $100 \mathrm{~nm}[1,2]$. Nanotechnology is considered an emerging technology due to the possibility to advance well-established products and to create new products with totally new characteristics and functions with enormous potential in a wide range of applications. In addition to various industrial uses, great innovations are foreseenin information and communication technology, in biology and biotechnology, in medicine and medical technology, in metrology, etc. Significant applications of Nano sciences and Nano engineering lie in the fields of pharmaceutics, cosmetics, processed food, chemical engineering, highperformance materials, electronics, precision mechanics, optics, energy production, and environmental sciences. Nanotechnology is an emerging and dynamic field where over 50,000 nanotechnology articles have been published annually worldwide in recent years, and more than 2,500 patents are filed at major patent offices such as the European Patent Office [3]. Nanotechnology can help in solving serious humanity problems such as energy adequacy, climate change or fatal diseases: "Nanotechnology" Alcatel-Lucent is an area which has highly promising prospects for turning fundamental research into successful innovations. Not only to boost the competitiveness of our industry but also to create new products that will make positive changes in the lives of our citizens, be it in medicine, environment, electronics or any other field. Nano sciences and nanotechnologies open up new avenues of research and lead to new, useful, and sometimes unexpected applications. Novel materials and new-engineered surfaces allow making products that perform better. New medical treatments are emerging for fatal diseases, such as brain tumours and Alzheimer's disease. Computers are built with Nano scale components and improving their performance depends upon shrinking these dimensions yet further" [4]. Nano materials with unique properties such as: nanoparticles carbon nanotubes fullerenes, quantum dots, quantum wires, Nano fibers, and Nano composites allow completely new applications to be found. Products containing engineered Nano materials are already in the market. The range of commercial products available today is very broad, including metals, ceramics, polymers, smart textiles, cosmetics, sunscreens, electronics, paints and varnishes. However new methodologies and instrumentation have to be developed in order to increase our knowledge and information on their properties. Nano materials must be examined for potential effects on health as a matter of precaution, and their possible environmental impacts.

\section{Major Fields of Research}

- Following are the major fields in which nanotechnology is being researched -

- Advance computing - Developing super computer

- Electronics - developing conductors and semiconductors

- Medicines - Developing technology to treat cancer (especially breast cancer)

- Textile Engineering - Nanofabrication, etc.

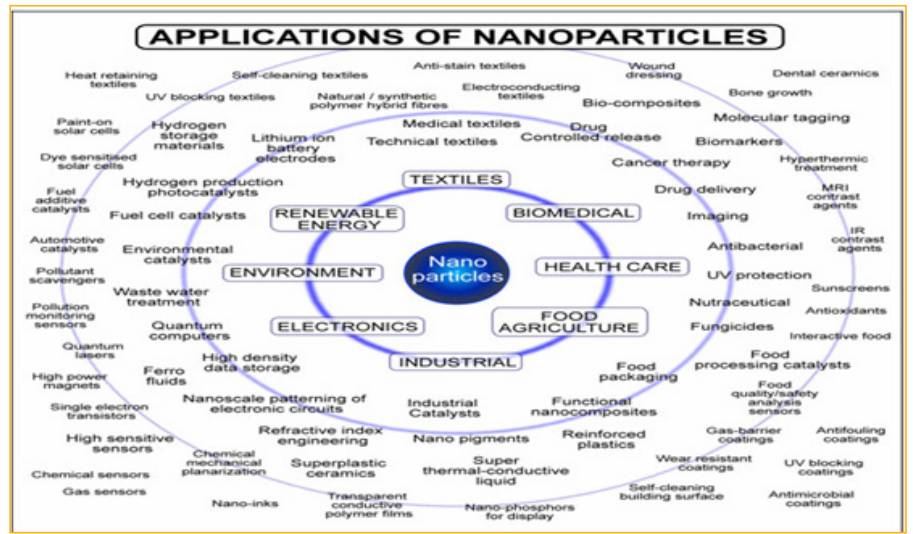




\section{History of Nanotechnology}

The development in the field of nanotechnology started in 1958 and the various stages of development have been summarized in Table 1.between 1 and 999 nanometers (1-999 nm) (Figure2)

\begin{tabular}{|c|c|}
\hline Year & Development in Nanotechnology \\
\hline 1959 & R. Feynmaninitiated thought process \\
\hline 1974 & The term nanotechnology was used by Taniguchi for the first time. \\
\hline 1981 & IBM Scanning Tunneling Microscope \\
\hline 1985 & "Bucky Ball" \\
\hline 1986 & $\begin{array}{l}\text { First book on nanotechnology Engines of Creation published by K. Eric Drexler, Atomic Force Mi- } \\
\text { croscope }\end{array}$ \\
\hline 1989 & IBM logo was made with individual atoms \\
\hline 1991 & S. Iijima discovered Carbon Nano tube for the first time. \\
\hline 1999 & 1st Nano medicine book by R. Freitas "Nano medicine" was published \\
\hline 2000 & For the first time National Nanotechnology Initiative was launched \\
\hline 2001 & $\begin{array}{l}\text { For developing theory of nanometer-scale electronic devices and for synthesis and characterization of } \\
\text { carbon nanotubes and Nano wires, Feynman Prize in Nanotechnology was awarded }\end{array}$ \\
\hline 2002 & $\begin{array}{l}\text { Feynman Prize in Nanotechnology was awarded for using DNA to enable the self-assembly of new } \\
\text { structures and for advancing our ability to model molecular machine systems. }\end{array}$ \\
\hline 2003 & $\begin{array}{l}\text { Feynman Prize in Nanotechnology was awarded for modeling the molecular and electronic struc- } \\
\text { tures of new materials and for integrating single molecule biological motors with nano-scale silicon } \\
\text { devices. }\end{array}$ \\
\hline 2004 & $\begin{array}{l}\text { First policy conference on advanced nanotech was held. First center for nano mechanical systems was } \\
\text { established, Feynman Prize in Nanotechnology was awarded for designing stable protein structures } \\
\text { and for constructing a novel enzyme with an altered function. }\end{array}$ \\
\hline $2005-2010$ & $\begin{array}{l}\text { 3D Nano systems like robotics, 3D networking and active nano products that change their state } \\
\text { during use were prepared. }\end{array}$ \\
\hline 2011 & Era of molecular nano technology started \\
\hline
\end{tabular}

Table 1: Periodical Development in Nanotechnology.

\section{Some Applications of Nanotechnology in Agriculture}

a. Crop improvement

b. Increase efficient fertilizers and pesticides

c. Soil management.

d. Plant disease detection.

e. Water management

f. Analysis of gene expression and Regulation

g. Post-Harvest Technology.

\section{Some Applications of Nanotechnology in Medicine}

One application of nanotechnology in medicine currently being developed involves employing nanoparticles to deliver drugs, heat, light or other substances to specific types of cells, such as cancer cells. Particles are engineered so that they are attracted to diseased cells, which allow direct treatment of those cells. This technique reduces damage to healthy cells in the body and allows for earlier detection of disease. For example, nanoparticles that deliver chemotherapy drugs directly to cancer cells are under development.

\section{Nano scale and Nanostructures}

The nano scale is the place where the properties of most common things are determined just above the scale of an atom. Nano scale objects have at least one dimension (height, length, depth) that measures between 1 and 999 nanometers $(1-999 \mathrm{~nm})$ (Figure 2) 


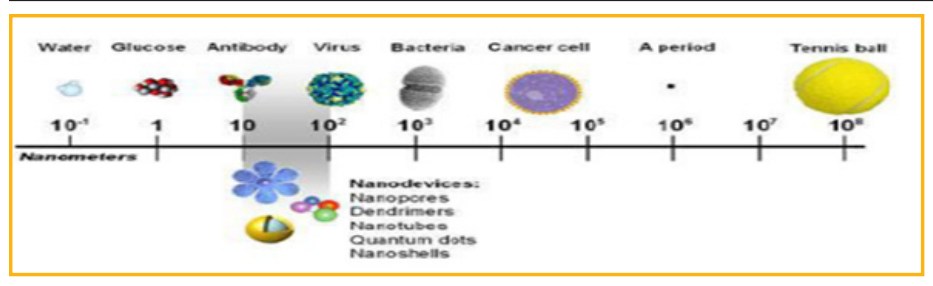

\section{Medical use of Nano Materials}

Nano medicine is a relatively new field of science and technology. By interacting with biological molecules at nano scale, nanotechnology broadens the field of research and application. Interactions of nano devices with bio molecules can be understood both in the extracellular medium and inside the human cells. Operation at nano scale allows exploitation of physical properties different from those observed at micro scale such as the volume/surface ratio.

\section{Some Applications of Nanotechnology in Electronics Nano electronics - Nanotechnology in Electronics}

The term nano electronics refers to the use of nanotechnology in electronic components. These components are often only a few nanometers in size. However, the tinier electronic components become, the harder they are to manufacture. Nano electronics covers a diverse set of devices and materials, with the common characteristic that they are so small that physical effects alter the materials' properties on a nano scale - inter-atomic interactions and quantum mechanical properties play a significant role in the workings of these devices. At the nano scale, new phenomena take precedence over those that hold sway in the macro-world. Quantum effects such as tunneling and atomistic disorder dominate the characteristics of these nano scale devices. The first transistors built in 1947 were over 1 centimeter in size; the smallest working transistor today is 7 nanometers long - over 1.4 million times smaller $(1 \mathrm{~cm}$ equals 10 million nanometers). The result of these efforts are billiontransistor processors where, once industry embraces $7 \mathrm{~nm}$ manufacturing techniques, 20 billion transistor-based circuits are integrated into a single chip.

\section{Nano Electronic Devices Spintronics}

Besides transistors, nano electronic devices play a role in data storage (memory). Here, spintronics - the study and exploitation in solid-state devices of electron spin and its associated magnetic moment, along with electric charge - is already an established technology. Read more: "Graphene spintronics - from science to technology". Spintronics also plays a role in new technologies that exploit quantum behavior for computing (read more: "Quantum computing moves forward with spintronics progress" and "The birth of topological spintronics").

\section{Optoelectronics}

Electronic devices that source, detect and control light - i.e. optoelectronic devices - come in many shapes and forms. Highly energy-efficient (less heat generation and power consumption) optical communications are increasingly important because they have the potential to solve one of the biggest problems of our information age: energy consumption. In the field of nanotechnology, materials like nano fibers (see for instance: "Light-emitting nano fibers shine the way for optoelectronic textiles") and carbon nanotubes have been used and especially graphene has shown exciting potential for optoelectronic devices.

\section{Displays}

Display technologies can be grouped into three broad technology areas; Organic LEDs, electronic paper and other devices intended to show still images, and Field Emission Displays. For more, read our special section on Nanotechnology in Displays.

\section{Wearable, flexible electronics}

The age of wearable electronics is upon us as witnessed by the fast growing array of smart watches, fitness bands and other advanced, next-generation health monitoring devices such as electronic stick-on tattoos. If current research is an indicator, wearable electronics will go far beyond just very small electronic devices or wearable, flexible computers. Not only will these devices be embedded in textile substrates but an electronics device or system could ultimately become the fabric itself. Electronic textiles (e-textiles) will allow the design and production of a new generation of garments with distributed sensors and electronic functions. Such e-textiles will have the revolutionary ability to sense, act, store, emit, and move - think biomedical monitoring functions or new man-machine interfaces - while ideally leveraging an existing low-cost textile manufacturing infrastructure (see for instance "wearing single-walled carbon nanotube electronics on your skin", a "temporary tattoo to monitor 
glucose levels" or "graphene nano sensor tattoo on teeth monitors bacteria in your mouth").

\section{Applications in Electronics}

The semiconductor industry has been able to improve the performance of electronic systems for more than four decades by downscaling silicon-based devices but this approach will soon encounter its physical and technical limits. This fact, together with increasing requirements for performance, functionality, cost, and portability have been driven the microelectronics industry towards the nano world and the search for alternative materials to replace silicon. Carbon nano materials such as one dimensional (1D) carbon nanotubes and two dimensional (2D) graphene have emerged as promising options due to their superior electrical properties which allow for fabrication of faster and more power-efficient electronics. Graphene transistor. In 2004, it was shown for the first time that a single sheet of carbon atoms packed in a honeycomb crystal lattice can be isolated from graphite and is stable at room temperature. The new nanomaterial, which is called graphene, allows electrons to move at an extraordinarily high speed. This property, together with its intrinsic nature of being one atom-thick, can be exploited to fabricate field-effect transistors that are faster and smaller. Carbon nanotube electronics. When a layer of graphene is rolled into a tube, a single-walled carbon nanotube (SWNT) is formed. Consequently, SWNTs inherit the attractive electronic properties of graphene but their cylindrical structure makes them a more readily available option for forming the channel in field-effect transistors. Such transistors possess an electron mobility superior to their silicon-based counterpart and allow for larger current densities while dissipating the heat generated from their operation more efficiently. During the last decade, carbon nano tubebased devices have advanced beyond single transistors to include more complex systems such as logic gates and radio-frequency components. Carbon-based nano sensors. In addition to the exceptional electrical properties of graphene and carbon nanotubes, their excellent thermal conductivity, high mechanical robustness, and very large surface to volume ratio make them superior materials for fabrication of electromechanical and electrochemical sensors with higher sensitivities, lower limits of detection, and faster response time. A good example is the carbon nanotubebased mass sensor that can detect changes in mass caused by a single gold atom.

\section{Some applications of Nanotechnology in Environment}

Nanotechnology is being used in several applications to improve the environment. This includes cleaning up existing pollution, improving manufacturing methods to reduce the generation of new pollution, and making alternative energy sources more cost effective. Potential applications include:

- Cleaning up organic chemicals polluting groundwater. Researchers have shown that iron nanoparticles can be effective in cleaning up organic solvents that are polluting groundwater. The iron nanoparticles disperse throughout the body of water and decompose the organic solvent in place. This method can be more effective and cost significantly less than treatment methods that require the water to be pumped out of the ground.

- Generating less pollution during the manufacture of materials. Researchers have demonstrated that the use of silver nano clusters as catalysys can significantly reduce the polluting byproducts generated in the process used to manufacture propylene oxide. Propylene oxide is used to produce common materials such as plastics, paint, detergents and brake fluid.

- Increasing the electricity generated by windmills. Epoxy containing carbon nanotubes is being used to make windmill blades. The resulting blades are stronger and lower weight and therefore the amount of electricity generated by each windmill is greater.

- Producing solar cells that generate electricity at a competitive cost. Researchers have demonstrated that an array silicon nanowires embedded in a polymer results in low-cost but high-efficiency solar cells. This may result in solar cells that generate electricity as cost effectively as coal or oil.

Some Applications of Nanotechnology in Consumer Products

Nanotechnology has already found its way into numerous consumer products you use every day, from clothing to skin lotion. They include:

- Silver nanoparticles in fabric that kill bacteria making clothing odor-resistant.

- Skin care products that use nanoparticles to deliver vitamins deeper into the skin

- Lithium ion batteries that use nanoparticle-based electrodes powering plug-in electric cars

- Flame retardant formed by coating the foam used in furniture with carbon nano fibers. 


\section{Nano Technological Applications in Food Industry}

Nano technology has been reported as the new industrial revolution, both developed, and developing countries are investing in this technology to secure a market share. Food processing approaches that involve nano materials include integration of nutraceuticals, gelation and viscosifying agents, nutrient propagation, mineral and vitamin fortification, and nano-encapsulation of flavors .Thus, systems with physical structures in the nanometer distance range could affect features from food safety to molecular synthesis. Nanotechnology may also have the potential to enhance food quality and safety. Many studies are assessing the ability of nano sensors to improve pathogen detection in food systems. Nano foods are products that were grown processed or packaged with the aid of nanotechnology or materials produced with nanotechnology (Fig. 1). In this review, we discuss some current nanotechnology research in food technology and agriculture, including processing, packaging, nano-additives, cleaning, and sensors for the detection of contaminants, and propose future developments in the developing field of agrifood nanotechnology (Figure. 2).

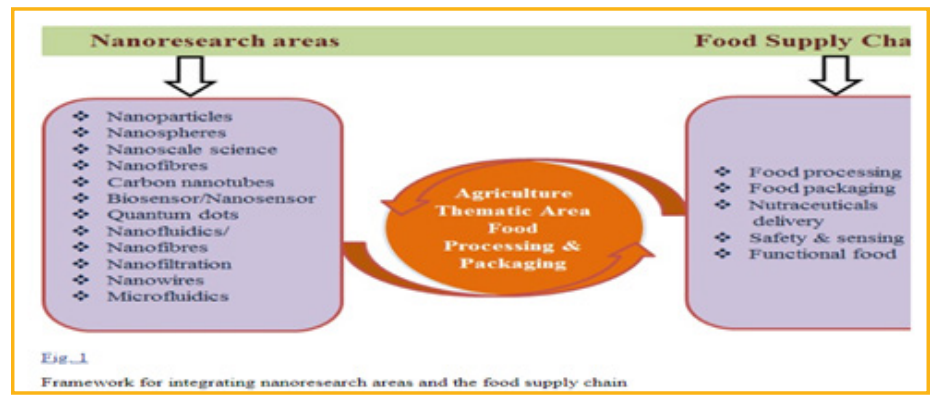

Figure 1: Framework Integrating Nanoresearch Areas and the Food Supply chain.

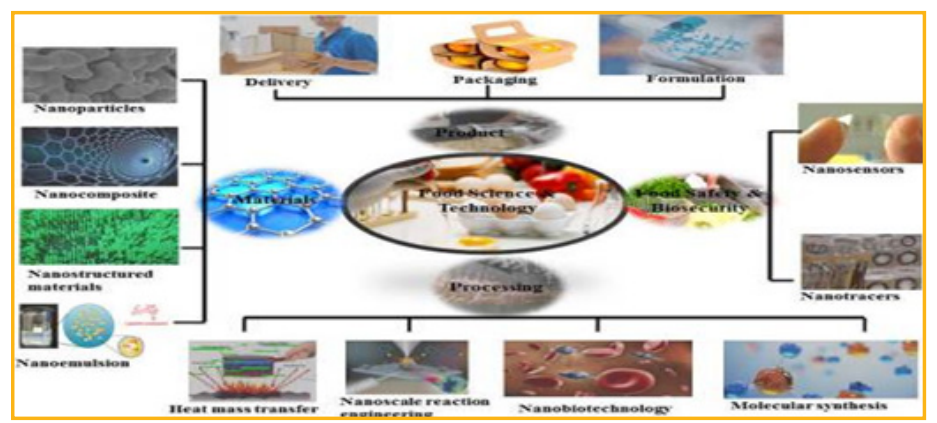

Figure 2: Developing fileds of Agrifood Nanotechnology.

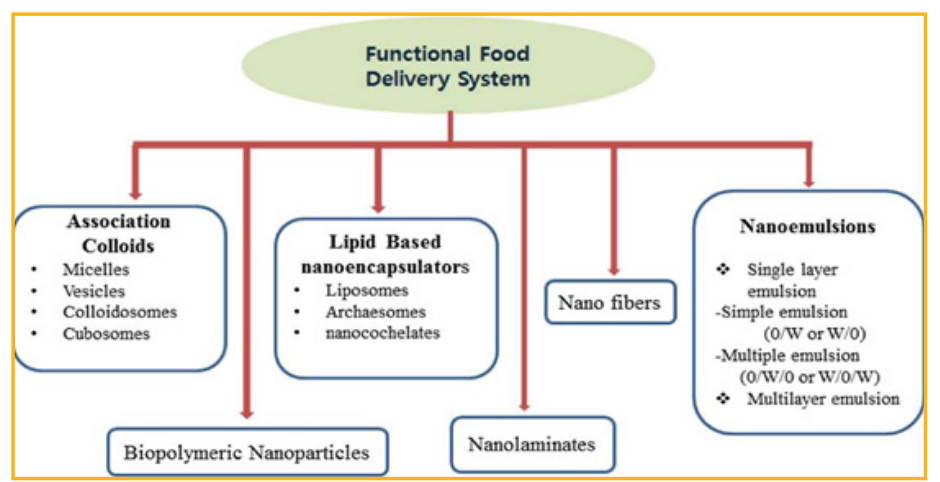

Benefits of Nano Materials in Food Packaging Uses

Bioactive-packaging materials can aid the oxidation of foodstuffs and avoid the development of off-flavors and unwanted textures. Non sustainable production, lack of recyclability, and insufficient mechanical and barrier properties are some of the ongoing challenges faced by the food and packaging industries. Although metal and glass are excellent barrier materials that can be used to inhibit undesirable mass transport in food packaging, plastics are still popular due to their lightweight, formability, cost effectiveness, and versatility. Indeed, the packaging industry accounts for more than $40 \%$ of all plastic usage, with half of this $40 \%$ used for food packaging revealed that the development of exciting novel nanotechnology products for food packaging, and some antimicrobial films had been introduced to increase the shelf-life of food and dairy products Moreover, food preservation and food packaging materials have become essential in the food industry. Food spoilage can be detected using nano sensors; thousands of nanoparticles fluoresce in several colors after coming into contact with food pathogens. In our studies of the significance of time in nourishment microbiology, the chief goal of nano sensors was to decrease the time for pathogen detection from days to hours or even minutes. Packaging prepared with nano sensors can also track either the internal or external circumstances of food products, vessels, and pellets. For example, Opel, which is used to make Opalfilm, containing 50-nm carbon black nanoparticles, was used as a biosensor that could change color in response to food spoilage [5-13]. 


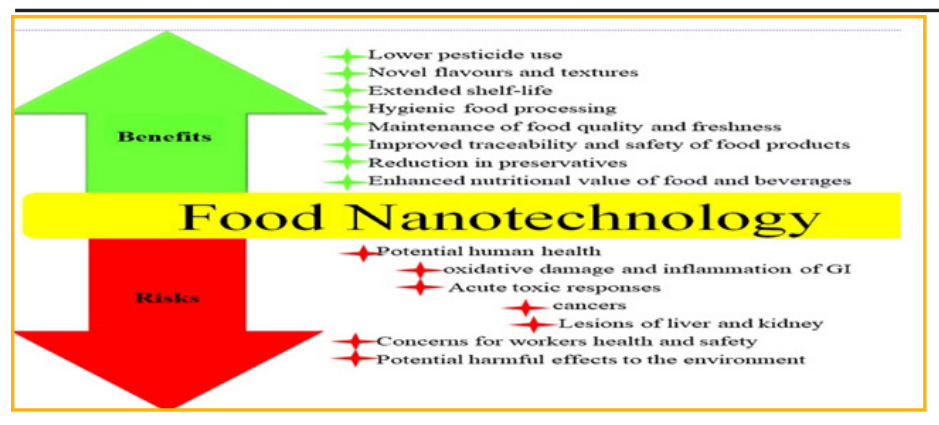

\section{Conclusion}

Nanotechnology follows biology and information technology. Nanotechnology development in order to quality of life. Nanotechnology will continue to develop the society and improve the environment in various ways. Today, thanks to nanotechnology, we can buy tennis racket that are stronger. So, future research in this field is necessary for its application.

\section{Acknowledgements}

Thanks to my parents. And my son Yash Gupta Finally, thanks to all the friends for their continued support and encouragement.

\section{References}

1. Nikalje AP (2017) Nanotechnology and its Applications in Medicine. Med chem5: 081-089.

2. Rasheed SAP, Jude M, Suresh K, Dey S, Sunil H, et al. (2019) Nanotechnologyand Its Applications in Dentistry. International Journal of Advanced HealthSciences 2: $1-10$.

3. Shrivastava S, Dash D (2013) Applying Nanotechnology to Human Health: Revolution in Biomedical Sciences. J Nanotech 1-15.

4. Deb KD, Griffith M, Muinck ED, Rafat M (2012) Nanotechnology in stem cellsresearch: advances and applications.Front Biosci (Landmark Ed) 17: 1747-1760.

5. Boisseau P, Loubaton B (2011) Nanomedicine, nanotechnology in medicine. ComptesRendus Physique 12: 620-636.

6. (2010) Nanotechnology in Targeted Cancer Therapy. University of Waterloo.

7. LaVan DA, McGuire T, Langer R (2010) Small-scale systems for in vivo drugdelivery. Nat Biotechnol 21: 1184-1191.

8. Singh K, Panghal M, Kadyan S, Yadav JP (2014)
Evaluation of Antimicrobial Activity of Synthesized Silver NanoparticlesusingPhyllanthus amarus and Tinospora cordifolia Medicinal Plants. JNanomed Nanotechnol 5: 250.

9. Connolly JM, Raghavan V, Owens P, Wheatley A, Keogh I, et al. (2014)Nanogold-based hotosensitizers Probes for Dual-model Bioimaging and Therapy of Cancer. J Nanomed Nanotechnol 5: 249.

10. Hsiao I, Gramatke AM, Joksimovic R, Sokolowski M, Gradzielski M, et al. (2014) Size and Cell Type Dependent Uptake of Silica Nanoparticles. J Nanomed Nanotechnol 5: 248.

11. Tiwari V, Khokar MK, Tiwari M, Barala S, Kumar M (2014) Anti-bacterial Activity of Polyvinyl yrrolidone Capped Silver Nanoparticles on theCarbapenem Resistant Strain of Acinetobacter baumannii. J Nanomed Nanotechnol 5: 246.

12. Baccar H, Mejri MB, Prehn R, del Campo RJ, Baldrich E, et al. (2014) Interdigitated Microelectrode Arrays Integrated in Microfluidic Cell for Biosensor Applications. J Nanomed Nanotechnol 5: 243.

13. Ramesh Kumar K, Nattuthurai, Gopinath P, Mariappan $\mathrm{T}$ (2014) Biosynthesis of Silver Nanoparticles from Morinda tinctoria Leaf Extract and their Larvicidal Activity against Aedes aegypti Linnaeus 1762. JNanomed Nanotechnol 5: 242.Ther 5:195.

Copyright: (92020 Sumit Kumar Gupta. This is an open-access article distributed under the terms of the Creative Commons Attribution License, which permits unrestricted use, distribution, and reproduction in any medium, provided the original author and source are credited. 